\title{
Patrones de urbanización en la biodiversidad de humedales urbanos en Concepción metropolitano ${ }^{1}$
}

\author{
Carolina Rojas², Einer Sepúlveda-Zúñiga3 ${ }^{3}$ Olga Barbosa ${ }^{4}$, \\ Octavio Rojas ${ }^{5}$ y Carolina Martínez ${ }^{6}$
}

\begin{abstract}
RESUMEN
La urbanización en Concepción metropolitano $\left(36^{\circ} \mathrm{S}\right)$ en las últimas décadas ha impactado áreas de valor ecológico, tales como humedales y lagunas. Aquí analizamos la perturbación de parámetros de biodiversidad en seis humedales urbanos, mediante la exploración y uso de indicadores. Se proponen tres indicadores: naturalidad y riqueza de macrolepidópteros y plantas, los que se evalúan mediante modelos de regresión múltiple. Los resultados indican que los humedales con menor grado de perturbación antrópica se encuentran ubicados dentro de una reserva protegida y rodeados de una matriz de paisaje seminatural; mientras que los más perturbados están más próximos a la urbanización. Además, la riqueza de macrolepidópteros se ve más afectada por la distancia a carreteras, en cambio la riqueza de plantas por la densidad de viviendas. Se genera una herramienta para la evaluación de efectos de la urbanización en humedales urbanos.
\end{abstract}

Palabras clave: Humedales, Urbanización, Biodiversidad, Naturalidad

\begin{abstract}
Urbanization of the Metropolitan region of Concepción $\left(36^{\circ} \mathrm{S}\right)$ in the last decades has impacted on the ecological value of areas such as wetlands and lagoons. Here, we analyse the disturbance to biodiversity indicators in six urban wetlands. Three measures are proposed: a naturalness indicator and two Macrolepidoptera plant indicators of species richness. Multiple regression models were developed for the assessment of indicators. Results show that the wetlands with the lowest degree of human interference are located in a protected area and surrounded by a matrix of semi-natural landscapes, whereas the more disturbed wetlands are those closer to areas of urbanization. Results indicate that macrolepidopterans richness is more affected by the distance to roads, in contrast with the richness of plants, which is affected by housing density. We generated a tool for the assessment of urbanization effects in urban wetlands.
\end{abstract}

Key words: Wetlands, Urbanization, Biodiversity, Naturalness

\footnotetext{
Este trabajo ha contado con el apoyo financiero de CONICYT a través de su programa FONDAP $\mathrm{N}^{\circ} 15110020$ y FONDECYT No 1150459. Agradecemos el apoyo de FONDECYT N¹1110183, CONICYT PFB-23 y VIRD No214.603.015-1.0. Artículo recibido el 17 de octubre de 2014, aceptado el 11 de mayo de 2015 y corregido el 22 de junio de 2015.

2 Departamento de Geografía, Universidad de Concepción (Chile). E-mail: crojasq@udec.cl
}

\footnotetext{
Departamento de Zoología, Universidad de Concepción (Chile). E-mail: einersepulveda@udec.cl

4 Instituto de Ciencias Ambientales y Evolutivas, Universidad Austral de Chile (Chile).

E-Mail: olga.barbosa@uach.cl

5 Departamento de Planificación Territorial y Sistemas Urbanos, Universidad de Concepción (Chile). E-Mail: ocrojas@udec.cl

6 Instituto de Geografía, Pontificia Universidad Católica de Chile (Chile). E-mail: camartinezr@uc.cl
} 
El crecimiento urbano de las ciudades es un fenómeno mundial acelerado, que ha producido transformaciones drásticas del paisaje en el siglo XX; como la alteración de terrenos seminaturales y agrícolas, el aumento de la tasa de consumo de recursos naturales como el agua, y la presión constante a la biodiversidad y provisión de servicios ecosistémicos (Vitousek, 1997; Niemelä et al., 2010). Estos efectos se intensifican con el fenómeno de "dispersión urbana" o "urban sprawl", cuyos patrones fomentan el incremento de la distancia de los asentamientos urbanos desde el centro de la ciudad, consumiendo suelo hacia lugares cada vez más periféricos, presionando espacios naturales frágiles (Muñiz \& Galindo, 2005), como humedales (Rojas et al., 2013a).

Los humedales, corresponden a biotopos de composición y estructura compleja, de delicado equilibrio ecológico, localizados en: zonas de transición entre sistemas acuáticos y terrestres, que sostienen vegetación hidrófita y mantienen substrato saturado de agua (Cowardin et al., 1979; Margalef, 1987 en: Viñals, 1999). Estos ambientes han sido utilizados por la población debido a los múltiples servicios ecosistémicos que entregan (e.g. provisión de hábitat, remoción de contaminantes, almacenaje de agua, mitigación de desastres socionaturales y regulación microclimática) (Bolund \& Hunhammar, 1999; Mitsch \& Gossenlink, 2007). En este sentido es común que asentamientos humanos se emplacen en sus bordes; lo cual genera una perturbación en su biodiversidad y capacidad de proveer estos beneficios a la comunidad. Por tanto su permanente pérdida por urbanización es una amenaza a la continuidad de provisión de estos servicios ecosistémicos en la ciudad (Barbosa \& Villagra, 2015).

En Chile más del $89 \%$ de la población vive en ciudades (UN, 2014), sin embargo el conocimiento sistematizado de los efectos de la urbanización sobre la biodiversidad de humedales ha sido escaso. Esto es particularmente preocupante para el caso de humedales situados dentro de los límites urbanos, debido a su complejidad ecológica y el desconocimiento en la valoración de estos (Gómez-Baggenthun \& Barton, 2013). A nivel nacional se han realizado los trabajos de Castro et al. (1993), los inventarios de la Comisión Nacional de Medio Ambiente CONAMA (1999, 2000 y 2005), el Ministerio de Medio Ambiente MMA (2011) y la Comisión Nacional de Medio Ambiente y el Centro de Ecología Aplicada CONAMA-CEA (2006), esta última clasifica los humedales nacionales en tres ecotipos principales: marinos, costeros y continentales. Desde los efectos de la antropización destacan los trabajos de Bertrán et al. (2010), Hauenstein et al. (2002 y 2005) y Peña et al. (2006). A pesar de estos esfuerzos, el deterioro de los humedales urbanos continúa, incluso la débil articulación con los instrumentos de planificación urbana para protegerlos y reconocer su importancia, ha resultado en una pérdida mayor en varias ciudades del país, principalmente en áreas costeras (Barbosa \& Villagra, 2015; Smith y Romero, 2009).

De este modo, la motivación principal de esta investigación es analizar cómo los patrones de urbanización afectan parámetros de biodiversidad en seis humedales urbanos, localizados en la costa de Concepción metropolitano (Región del Biobío), zona donde existen numerosos humedales con un valioso patrimonio ambiental (González y Victoriano, 2005). Los parámetros de biodiversidad son analizados utilizando tres indicadores: (i) riqueza de especies de macrolepidópteros, (ii) riqueza de especies de plantas y (iii) las características del uso de suelo determinadas con un indicador de naturalidad. Posteriormente estos indicadores son evaluados mediante modelos de regresión múltiple. Se espera que las comunidades de macrolepidópteros y plantas, respondan de forma diferencial a los atributos de la matriz del hábitat y a las variables de urbanización.

Su novedad radica en primer lugar en la cobertura geográfica, se analizan humedales muy reconocidos por el impacto de la urbanización, como Rocuant-Andalién y Lenga (Pauchard et al., 2005; Smith y Romero, 2009); y otros como Laguna Verde, San Andrés, Paicaví y Sector Cuatro Esquinas, en los cuales recientemente se ha comprobado la alteración de su diversidad biológica por efectos del suelo urbanizado (Sepúlveda et al., 2012). El estudio es un complemento a la investigación de Smith y Romero (2009) la cual se centra en la pérdida de calidad ambiental en los humedales Rocuant-Andalién y 
Lenga, entre otros; y a Pauchard et al. (2005), quienes evalúan los efectos de la dispersión urbana en la pérdida de hábitat en flora y fauna. Sin embargo, esta investigación no mide la pérdida de superficie de los humedales por cambios de usos de suelo a urbano como los trabajos anteriormente mencionados. El estudio continúa en la línea de investigación en biodiversidad de humedales propuesta en Sepúlveda et al. (2012); nuevamente se utiliza el indicador de naturalidad desarrollado por Rojas et al. (2013b), pero esta vez como una variable dependiente, ya que en esta oportunidad se profundiza en la influencia de una serie de variables urbanas en la biodiversidad de cada humedal. El estudio pretende aportar a la investigación geográfica que identifica la pérdida de biodiversidad por causa de la urbanización, más aún en una ciudad latinoamericana de rápido y continuo crecimiento urbano (Pauchard et al., 2005; Rojas et al., 2013a).

\section{Urbanización de humedales en áreas costeras}

Las áreas costeras son sistemas complejos e integrados; en general referidas a escalas espaciales de cientos de kilómetros y escalas temporales desde centenares de años a milenios (Sherman \& Bauer, 1993), presentando dificultades en su delimitación natural. Esto explica a su vez el carácter de la mayoría de las definiciones al asociarse con "aquel espacio en el cual los ambientes terrestres influencian los ambientes marinos y viceversa" (Carter, 1988; Woodroffe, 2003). La mayoría de las zonas costeras en el mundo, son resultado de procesos tectónicos y/o eustáticos con herencias morfogenéticas que condicionan su funcionamiento actual, generando una alta fragilidad ambiental cuando son objeto de urbanización y usos incompatibles (Isla et al., 2012).

La urbanización de las áreas costeras, implica una concentración de población y una progresiva implementación de nuevas actividades e infraestructuras. Este proceso ha transformado el paisaje, presionando fuertemente el suelo, incluso con megaciudades de más de 10 millones de habitantes (e.g. Mumbai, Río de Janeiro, etc.), por el atractivo de sus recursos económicos, medioambientales y potenciales usos recreacionales y turísticos (Sekovski et al., 2011). Se estima que prácticamente el $50 \%$ de la población mundial urbana (unos tres billones de personas), vive a menos de $60 \mathrm{~km}$ de la costa (Woodroffe, 2003), además estas ciudades poseen densidades poblacionales 2,6 veces mayor en comparación a zonas continentales interiores (MEA, 2005).

La urbanización de la costa tiene importante consecuencias como: homogenización y degradación del paisaje, alteración de procesos naturales como los flujos de nutrientes y energía, degradación de hábitats, pérdida de biodiversidad, disminución del patrimonio natural y cultural entre otros, cuyas consecuencias generan alteración en las funciones ecosistémicas, que se asocian a conflictos ambientales y socioculturales (Sekovski, 2011), los cuales se contraponen a modelos de desarrollo sustentable. Estos efectos, se han visto especialmente evidenciados en países mediterráneos; por ejemplo en España en los primeros $10 \mathrm{~km}$ de costa, se concentra un $30 \%$ de las áreas artificiales construidas del país, bajo el amparo de un desarrollo basado en la construcción y el boom inmobiliario (OSE, 2010).

Los esfuerzos para comprender y relacionar el funcionamiento del área costera con el proceso de urbanización se focalizan en dos áreas: la conservación biológica y los estudios de remediación y restauración. La conservación biológica ha estado principalmente enmarcada en los lineamientos de la Convención Ramsar de humedales (1971) (Borja et al., 2006; Rodrigues et al., 2011; Marques et al., 2013; Campuzano et al., 2013). Sin embargo, la tendencia de esta década ha sido el incorporar conceptos como remediación y restauración, así en Elliot et al. (2007) se propone su aplicación en ambientes costeros para acercarse a estados previos a la degradación. Por otro lado, mediante el manejo integrado de la zona costera (GIAL o MIZC) se buscan acciones efectivas para un desarrollo urbano más armónico con un enfoque sistémico (Cendrero et al., 2003; Barragán, 2003; Chen et al., 2005; Seingier et al., 2011; McLachlan et al., 2013).

Como consecuencia de lo anterior, entre los ambientes costeros más afectados por la 
progresiva urbanización se encuentran los humedales, que corresponden a importantes fuentes de diversidad biológica, producto de la alta heterogeneidad estructural que presentan (MacArthur \& Wilson, 1967; Collins \& Kuehl, 2000). Se encuentran entre los ecosistemas más importantes del planeta por su rol en los ciclos biogeoquímicos, incluyendo el hidrológico (Mitsh \& Gosselink, 2000) y el de carbono (Adame et al., 2013). Asimismo, son esenciales en la provisión de diversos servicios ecosistémicos (Koch et al., 2009). En este sentido los humedales insertos en áreas urbanas y periurbanas, ofrecen regulación microclimática local atenuando las islas de calor, depuración de contaminantes, drenaje de aguas lluvia, mitigación de procesos erosivos y espacios para la recreación (Bolund \& Hunhammar, 1999; MEA, 2005; Moreno et al., 2010). Por su alta fragilidad se degradan fácilmente con intervenciones antrópicas (e.g. rellenos, acumulación de basuras y escombros, entre otras). El resultado es un deterioro profundo de los ciclos de nutrientes y energía, disminución en la diversidad de especies y aumento de especies tolerantes a la contaminación (Ramírez et al., 2002; Cvetkovic \& Chow-Fraser, 2011); lo que afecta principalmente servicios ecosistémicos asociados al ciclo hidrológico y recreativos (Barbosa \& Villagra, 2015).

En Concepción metropolitano $\left(36^{\circ}-38^{\circ}\right.$ S), los humedales urbanos presentan una herencia morfogenética vinculada a la antigua Ilanura fluvio-deltaica del río Biobío (Ilabaca, 1989; Isla et al., 2012). Las investigaciones han destacado principalmente su funcionamiento dinámico en catastros florísticos o faunísticos con fines de conservación, destacando a Stuardo \& Valdovinos (1989), Constabel (1993), Quintana (1993), San Martín et al. (1992), Valdovinos et al. (1993), Stuardo et al. (1993), Jiménez (1999), CONAMA (1999), Carrasco (2003), Villagrán et al. (2006), González \& Victoriano (2005), Valdovinos (2006). Además corresponden a áreas de refugio y reproducción de aves silvestres (Quezada, 1986; Quintana, 1993; Espinoza, 1997). A pesar de la documentada biodiversidad, actualmente son amenazados por actividades como: rellenos, desecamiento, destrucción de sus formaciones vegetacionales, acumulación de basuras y escombros y contaminación de sus aguas (Ramírez et al., 2002). De acuerdo a los antecedentes en Concepción metropolitano más del 23\% (1.734 ha) de la superficie ocupada por humedales se ha perdido en las últimas tres décadas, producto de la acelerada urbanización, resultando humedales con distintos grados de fragmentación, pérdida y alteración del hábitat (Pauchard et al., 2005).

\section{Metodología}

El estudio corresponde a seis humedales urbanos: (1) Laguna Verde (9,9 ha); (2) Lenga (480,4 ha); (3) Sector Cuatro Esquinas (24,3 ha); (4) Rocuant-Andalién (1183,4 ha); (5) San Andrés (Parque Central) $(44,9$ ha) y (6) Paicaví (Lorenzo Arenas) (24,9 ha) (Figura № 1). Estos se localizan en el sector norte de Concepción metropolitano $\left(36^{\circ} \mathrm{S}\right)$, específicamente en la conurbación de las comunas de Concepción, Talcahuano y Hualpén. La zona se caracteriza por poseer una gran variedad de ecosistemas tanto fluviales como lacustres (Valdovinos, 2006) y ser única por su riqueza de lagunas y humedales (Parra, 1989).

De los humedales estudiados, cuatro corresponden a sistemas palustres (Laguna Verde, San Andrés, Paicaví y Sector Cuatro Esquinas), ya que están formados por pantanos de agua dulce, además tienen un origen fluvial a partir de la depresión del paleocauce formado por el río Biobío (Parra et al., 2004). Mientras que Lenga y Rocuant-Andalién son considerados humedales de tipo marisma, ya que presentan aguas salobres.

\section{Identificación humedales}

La identificación del perímetro de borde de los seis humedales urbanos, se realiza mediante clasificación supervisada de máxima probabilidad sobre una imagen satelital Landsat TM $(30 \mathrm{~m}$ ) del año 2010 (18 de marzo de 2010), se utiliza el clasificador Maximun Likelihood o máxima probabilidad (Rojas et al., 2013a). La delimitación de este perímetro fue validada en terreno y verificada mediante fotointerpretación de imágenes satelitales de alta resolución (Quickbird $2.4 \mathrm{~m}$ ), disponibles en Google Earth. Posteriormente se delimita la zona buffer de $1 \mathrm{~km}$. Asimismo, la matriz de cada uno de los humedales se define como el perímetro de borde y un área de influencia de $1 \mathrm{~km}$ (Figura $\mathrm{N}^{\circ} 1$ ). 
Figura $N^{\circ} 1$

Área de estudio: ubicación de los humedales y buffers de $1 \mathrm{~km}$ de los humedales

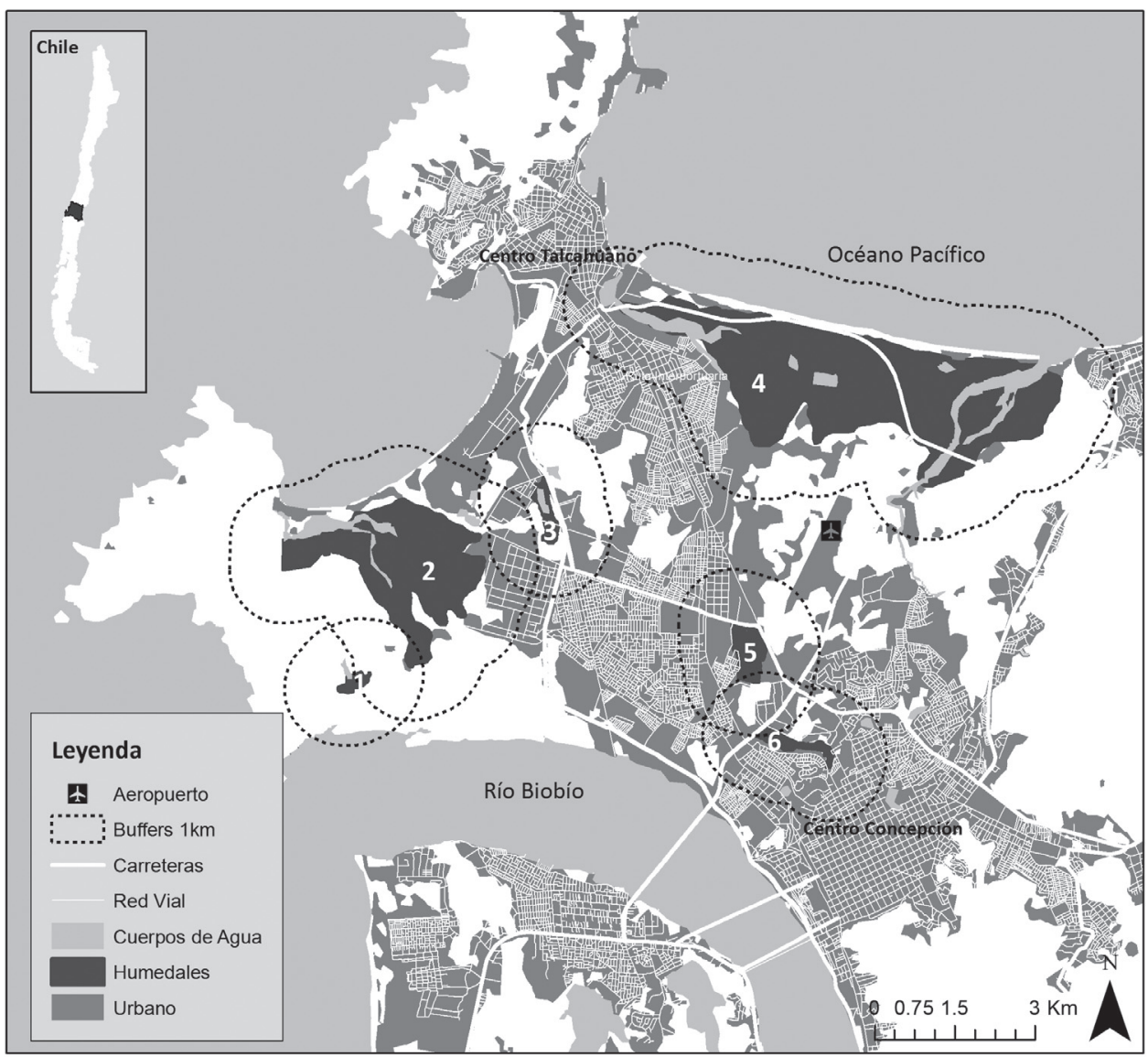

Nota: (1) Laguna Verde; (2) Lenga; (3) Sector Cuatro Esquinas ; (4) Rocuant-Andalién; (5) San Andrés (Parque Central); (6) Paicaví (Lorenzo Arenas).

Fuente: Elaboración propia.

\section{Indicadores de biodiversidad y naturalidad}

1) Riqueza de especies de plantas

Se define como primer indicador de biodiversidad debido a que las principales características topográficas, geológicas y estructurales de un ecosistema, están definidas generalmente por la diversidad y riqueza de plantas que lo conforman. La composición y estructura de las comunidades vegetales es una base fundamental para determinar el estado de conservación actual del área y el potencial cambio en los procesos de restauración o conservación a seguir (Gallo y Rodríguez, 2010).
Para determinar la riqueza vegetacional de cada humedal, se utilizan dos transectas de $30 \mathrm{~m}$ de largo, en las cuales se ubican 10 cuadrantes de $1 \mathrm{~m}^{2}$ (Figura $\mathrm{N}^{\circ} 2$ ). Las transectas por humedal se distribuyen de la siguiente forma:

i) Laguna Verde: transecta 1 se ubica a 140 $\mathrm{m}$ del centro del humedal y $980 \mathrm{~m}$ de la zona urbana, transecta 2 a $80 \mathrm{~m}$ al centro del humedal y $870 \mathrm{~m}$ de la zona urbana más cercana;

ii) Lenga; transecta 1 se ubica $1.000 \mathrm{~m}$ del centro del humedal y $260 \mathrm{~m}$ de zona urbana, transecta 2 a $1000 \mathrm{~m}$ del centro del humedal y $890 \mathrm{~m}$ de la zona urbana más cercana; 
iii) Sector Cuatro Esquinas: transecta 1 se ubica a $500 \mathrm{~m}$ del centro del humedal y 20 $\mathrm{m}$ de la zona urbana, transecta 2 a $300 \mathrm{~m}$ del centro del humedal, $20 \mathrm{~m}$ de la zona urbana más cercana;

iv) Rocuant-Andalién: transecta 1 se ubica a $1.000 \mathrm{~m}$ del centro del humedal y a 100 $\mathrm{m}$ de la zona urbana, transecta 2 a 1.500 $\mathrm{m}$ del centro del humedal y $100 \mathrm{~m}$ de la zona urbana más cercana;

v) San Andrés: transecta 1 se ubica a $350 \mathrm{~m}$ del centro del humedal y $30 \mathrm{~m}$ de la zona urbana, transecta 2 a $250 \mathrm{~m}$ del centro del humedal y $130 \mathrm{~m}$ de la zona urbana más cercana;

vi) Paicaví (Lorenzo Arenas). transecta 1 se ubica a $400 \mathrm{~m}$ del centro del humedal y a $30 \mathrm{~m}$ de la zona urbana, transecta 2 a $150 \mathrm{~m}$ al centro del humedal y $10 \mathrm{~m}$ de la zona urbana más cercana.

En cada transecta se determina la cobertura de las especies de plantas palustres emergidas, observando cuánto del área del cuadrante es ocupada por cada especie de planta. Para la identificación de las distintas especies vegetales, se colectan muestras de material para su herborización y posterior identificación mediante material bibliográfico (Matthei, 1995; Hoffmann, 1998; Riedemann y Aldunate, 2003; 2004; San Martín et al., 2006) y comparación directa con ejemplares depositados en el herbario de la Universidad de Concepción. Algunos taxa no fueron posibles de clasificar a nivel de especie debido a la ausencia de órganos de valor taxonómico (flores y/o frutos).

2) Riqueza de especies del grupo macrolepidóptera

Un segundo indicador de biodiversidad corresponde a la riqueza de especies de lepidópteros, estos constituyen uno de los grupos más utilizados como indicador del estado de conservación de la biodiversidad (Brown, 1997; Dufrene \& Legendre, 1997; Kremen, 1994; Andrade-C, 1998), siendo los macrolepidópteros aquellos de mayor tamaño dentro de este orden. Históricamente han sido usados como indicadores porque reúnen las siguientes características: taxonomía bien conocida, buen conocimiento de su ecología e historia natural, de fácil observación en el campo, amplitud tanto en el rango de ocupa- ción de hábitats como de rango geográfico, alta fidelidad ecológica y patrones biológicos relacionados con otros taxa (Andrade-C, 1998).

Se efectúa un muestreo mensual en cada humedal, dentro de los meses de octubre, noviembre y diciembre del año 2010, para los humedales Laguna Verde, San Andrés y Paicaví (datos obtenidos de Sepúlveda et al., 2012), estos puntos se distribuyen a una distancia media de las zonas urbanas de 900 m, 320 m y 55 m respectivamente. Para los humedales de Cuatro Esquinas, Lenga y Rocuant-Andalién, se realiza un muestreo en el mes de enero de 2011, con puntos a una distancia media de 15 m, 26 m y 280 m respectivamente (Figura $\mathrm{N}^{\circ} 2$ ).

Para la captura de macrolepidópteros nocturnos que poseen fototropismo positivo (Márquez, 2005), se utilizan 3 trampas de luz UV (Robinson \& Robinson, 1950; Frost, 1952) tipo Pennsylvania (Frost, 1957; Hollingsworth et al., 1963), distribuidas aleatoriamente en el borde de los humedales, con una distancia aproximada entre ellas de $100 \mathrm{~m}$, durante 5 horas comenzando desde la puesta de sol.

\section{Indicador de naturalidad}

Se define como un indicador morfométrico que varía de 0 a 1 . Mide el grado de perturbación e intervención del humedal, según el nivel de antropización y urbanización de los usos y coberturas del suelo localizados en la matriz de 1 km (Rojas et al., 2013b). Se formula con la siguiente ecuación que propone una relación entre las categorías de usos y coberturas del suelo y su ocupación en el año 2010 (Cuadro $N^{\circ} 1$ ).

$$
I N=\sum_{i=1}^{n} C S i * G N i / S T
$$

Donde: $\mathrm{CS}=$ Superficie total de cada una de las coberturas de suelo $i, \mathrm{GN}=$ Grado de Naturalidad definido para cada cobertura $i$ y $\mathrm{ST}=$ Superficie total del tipo de cobertura.

Las coberturas del suelo utilizadas corresponden a 10 capas temáticas de un mapa raster de resolución espacial $30 \mathrm{~m}$, detectadas a partir de las técnicas de interpretación de imágenes satelitales Landsat de marzo de 
Figura $\mathrm{N}^{\circ} 2$

Mapas transectas y puntos de muestreo
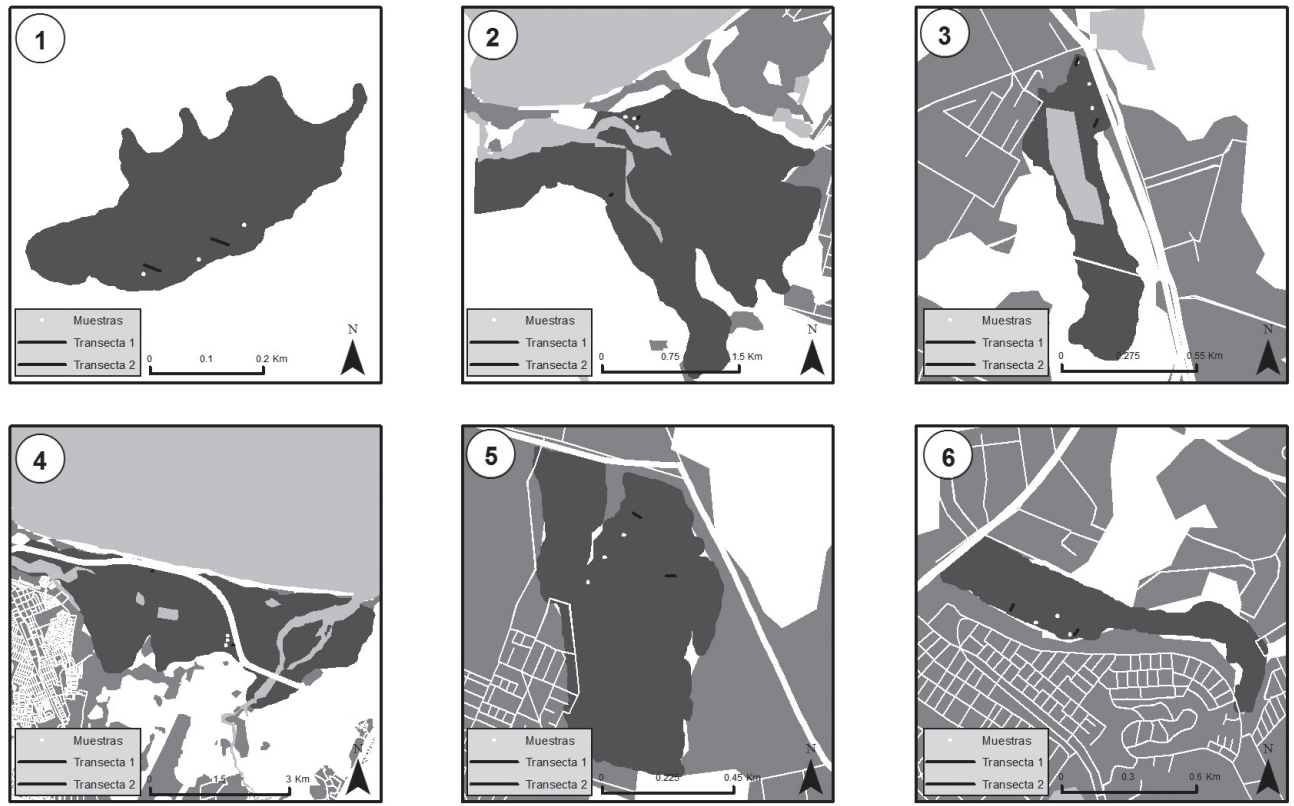

Nota: (1) Laguna Verde; (2) Lenga; (3) Sector Cuatro Esquinas; (4) Rocuant-Andalién; (5) San Andrés; (6) Paicaví. Fuente: Elaboración propia.

Cuadro $\mathrm{N}^{\circ} 1$

Los grados de naturalidad (gn) o nivel de perturbación

\begin{tabular}{|l|r|l|r|}
\hline Cobertura de Suelo & $(\mathrm{GN})$ & Cobertura de Suelo & $(\mathrm{GN})$ \\
\hline 1= Superficie Construida & 0 & 7= Playa Sedimentos & 0,5 \\
2= Bosque Nativo & 1 & 8= Praderas & 0,5 \\
3= Matorral & 0,75 & 9 = Terrenos Agrícolas & 0,25 \\
4= Cuerpos de Agua & 0,5 & $10=$ Humedales & 1 \\
5= Suelo Descubierto & 0,25 & 11 = No Data & - \\
6=Plantaciones Forestales & 0,25 & & \\
\hline
\end{tabular}

Fuente: Elaboración propia, modificado de Rojas et al. (2013b).

2010, mencionadas anteriormente. Los grados de naturalidad (GN) o nivel de perturbación se detallan en el Cuadro $\mathrm{N}^{\circ} 1$.

\section{Modelos estadísticos}

Para evaluar la relación entre las variables respuesta (a) riqueza de plantas, (b) riqueza de macrolepidópteros e (c) indicador de naturalidad, con la reciente urbanización, se utilizan tres modelos de regresión múltiple aplicados en el programa estadístico SPSS versión 17.0. Para cada variable respuesta, se manipulan siete (7) variables predictoras correspondientes a factores geográficos conductores de urbanización y cambios de usos 
de suelo, cuyos valores pueden observarse en el Cuadro $N^{\circ} 5$.

Las variables de urbanización son las siguientes:

- Distancia a carreteras (DC): Variable de accesibilidad, al igual que la superficie construida, es un indicador del nivel de urbanización, por tanto de perturbación del espacio natural, generalmente se ha utilizado en la modelización del crecimiento urbano (Aguayo et al., 2007; AlAhmadi et al., 2008; Rojas et al., 2014). Se calcula la distancia euclidiana a la carretera más cercana según cobertura del Ministerio de Obras Públicas MOP año 2010.

- Porcentaje Superficie urbanizada (S.U1): Representa el porcentaje de superficie construida, es un factor conductor de la pérdida de biodiversidad (Pauchard et al., 2005). Influye directamente en la pérdida de superficies y naturalidad de los humedales en el buffer $1 \mathrm{~km}$, por ejemplo en el estudio de Smith \& Romero (2009) se demostró el deterioro del humedal RocuantAndalién por avance de la construcción. Se extrae del mapa de coberturas de suelo de Rojas et al. (2013a).

- Porcentaje de Superficie urbanizable (S.U2): Variable de planificación que incluye el efecto de la urbanización futura, se ha utilizado como factor explicativo y predictivo del crecimiento urbano (Plata, et al., 2010; Rojas et al., 2014). Se extrae del mapa de expansión urbana desarrollado en Rojas et al. (2013b).

- Densidad de población (DP): Es una de las variables más reconocidas de urbanización, normalmente se utiliza en modelos explicativos y predictivos del crecimiento urbano (Aguayo et al., 2007: Rocha, et al., 2010; Rojas et al., 2014). Se calcula a nivel de manzana según datos del censo 2002.

- Densidad de viviendas (DV): Al igual que la densidad es una de las variable más reconocidas de urbanización, normalmente se utiliza en modelos explicativos del crecimiento urbano (Rojas et al., 2014). Se calcula a nivel de manzana según datos del censo 2002.

- Número de usos de suelo (NU): Variable que incorpora la diversidad de usos de suelo, mientras mayor es el número de usos en el buffer de $1 \mathrm{~km}$, existirá una mayor diversidad de coberturas. Generalmente se utiliza el índice de Shannon (Sepúlveda et al., 2012), pero se optó por una medida más simple. Se extrae del mapa de coberturas de suelo desarrollado en Rojas et al. (2013a).

- Índice de vegetación normalizada (NDVI): Se relaciona al uso de suelo y a la presencia de cobertura vegetacional, un índice cercano a 0 indica una menor vigorosidad en la vegetación, al contrario un índice más cercano a 1 un mayor vigor, se ha utilizado en la detección de la vegetación en humedales (Yuan et al., 2014; Vásquez, 2013). Se calcula desde la imagen satelital Landsat TM, mencionada anteriormente.

Para observar la autocorrelación se calcula el coeficiente de correlación de Pearson, posteriormente se aplica un análisis de componentes principales para utilizar solo las variables urbanas significativas que explican el total de la varianza. Los modelos estadísticos permiten observar el nivel de incidencia de las variables de urbanización seleccionadas en el entorno del humedal de $1 \mathrm{~km}$.

\section{Biodiversidad y urbanización}

Los humedales Laguna Verde, Paicaví, San Andrés y Cuatro Esquinas (humedales palustres de agua dulce) presentaron distintos grados de heterogeneidad vegetacional, pero coincidieron en especies de plantas helófitas dominantes, las cuales en orden de abundancia resultaron ser: Schoenoplectus californicus (C. A. Mey.) Soyák, seguida por Cyperus eragrostis, ambas de la familia Cyperaceae; otra representante de esta familia es Carexriparia Curt., muy abundante en los humedales Paicaví y San Andrés (Cuadro No 2). Cabe destacar que Laguna Verde se caracterizó por la presencia de Juncus procerus E. Mey., planta nativa perenne de hasta un metro de alto, que crece formando grandes champas y distribuyéndose en ambientes con anegamiento prolongado, pero no permanente (Añasco, 1981).

Los humedales Rocuant-Andalién y Lenga (humedales tipo marisma) presentaron un escaso número de especies, que puede ser relacionado con la elevada salinidad de estos 
ecosistemas, lo cual determinó una vegetación poco diversa y bastante homogénea. La Poaceae nativa Spartina densiflora Brongn., dominó en ambos humedales formando praderas o espartales, también fue posible encontrar, en algunos sectores la halófita suculenta Sarcocornia fruticosa (L.) Scott., observándose en muchos casos una combinación de ambas especies.

Cuadro $\mathrm{N}^{\circ} 2$

Composición taxonómica, porcentaje de cobertura y origen de las especies de plantas encontradas en los humedales palustres del gran concepción

\begin{tabular}{|c|c|c|c|c|c|c|c|c|}
\hline Familia & Especie & Origen & L.V. & P. & S.A. & R.A. & $\mathrm{L}$. & C.E. \\
\hline Equisetaceae & Equisetum giganteum $\mathrm{L}$. & $\mathrm{N}$ & 0 & 0,1 & 0 & 0 & 0 & 4 \\
\hline Alismataceae & Alisma lanceolatum With. & I & 0 & 0,01 & 0 & 0 & 0 & 0 \\
\hline Chenopodiaceae & Sarcocornia fructicosa (L.) Scott. & $\mathrm{N}$ & 0 & 0 & 0 & 22 & 28 & 0 \\
\hline Convolvulaceae & Convolvulus arvensis $\mathrm{L}$. & I & 0 & 0 & 0 & 0 & 0 & 3 \\
\hline \multirow[t]{5}{*}{ Cyperaceae } & Carex riparia Curt. & N & 0 & 26 & 10 & 0 & 0 & 0 \\
\hline & Cyperus eragrostis Lam. & N & 7 & 23 & 20 & 0 & 0 & 0 \\
\hline & Cyperus sp. & $\mathrm{N}$ & 0 & 0 & 0 & 0 & 0 & 42 \\
\hline & Schoenoplectus sp. & $\mathrm{N}$ & 0 & 0 & 0 & 0 & 0 & 11,25 \\
\hline & $\begin{array}{l}\text { Schoenoplectus californicus (C.A. } \\
\text { Mey.) Soyák }\end{array}$ & $\mathrm{N}$ & 36 & 30 & 30 & 0 & 0 & 7,25 \\
\hline Juncaceae & Juncus procerus E. Mey. & $\mathrm{N}$ & 44 & 0 & 8 & 20 & 0 & 0 \\
\hline \multirow[t]{6}{*}{ Poaceae } & $\begin{array}{l}\text { Paspalum paspalodes (Michx) } \\
\text { Scribner }\end{array}$ & I & 0 & 0,1 & 15 & 0 & 0 & 0 \\
\hline & Agrostis sp. & I & 0 & 0 & 0 & 0 & 0 & 0 \\
\hline & Spartina densiflora Brongn. & N & 0 & 0 & 0 & 28,82 & 51 & 0 \\
\hline & Bromus catharticus Vahl & I & 0 & 0 & 0 & 0 & 0 & 0 \\
\hline & Poa sp1 & 1 & 11 & 12 & 0 & 0 & 0 & 0 \\
\hline & Poa sp2 & I & 0 & 0 & 0 & 20 & 10 & 10 \\
\hline Typhaceae & Thypa dominguensis Pers. & $\mathrm{N}$ & 0 & 1 & 0 & 0 & 0 & 0 \\
\hline \multirow[t]{4}{*}{ Asteraceae } & Baccharis sagittalis (Less.) D.C. & $\mathrm{N}$ & 0 & 0 & 2 & 0 & 0 & 0 \\
\hline & Bidens laevis(L.) B.S.P & I & 0 & 0 & 0 & 0 & 0 & 21 \\
\hline & Cotula coronopifolia $L$ & 1 & 0 & 0 & 0 & 9 & 11 & 0 \\
\hline & Anthemis cotulaL. & I & 0 & 0 & 0 & 0 & 0 & 0 \\
\hline \multirow[t]{2}{*}{ Fabaceae } & Trifolium sp & I & 0 & 2 & 0 & 0 & 0 & 0 \\
\hline & Galega officinalisL. & I & 0 & 0 & 0 & 0 & 0 & 0,43 \\
\hline Hidrocotylaceae & Hydrocotyle ranunculoides L. f. & I & 0 & 0,4 & 0 & 0 & 0 & 0 \\
\hline \multirow[t]{3}{*}{ Polygonaceae } & Polygonum persicaria L. & I & 2 & 5 & 6 & 0 & 0 & 1 \\
\hline & Rumex acetosellaL. & 1 & 0 & 0 & 0 & 0 & 0 & 0 \\
\hline & Rumex crispusL. & I & 0 & 0,1 & 9 & 0,18 & 0 & 0 \\
\hline \multirow[t]{2}{*}{ Brassicaceae } & Sisymbrium officinaleL. & I & 0 & 0 & 0 & 0 & 0 & 0 \\
\hline & Raphanus sativusL. & I & 0 & 0 & 0 & 0 & 0 & 0 \\
\hline Apiaceae & Daucus carota L. & I & 0 & 0 & 0 & 0 & 0 & 0 \\
\hline
\end{tabular}

Nota: L.V.: Laguna Verde; P.: Paicaví; S.A.: San Andrés; R.A.: Rocuant Andalién; L.:Lenga; C.E.: Cuatro Esquinas. Fuente: Elaboración propia. 


\section{Macrolepidópteros}

Laguna Verde fue el humedal que presentó los valores más altos de diversidad de Macrolepidópteras; además se encontró la mayor abundancia de la familia Geometridae y fue el único humedal en el que se encontra- ron representantes de las familias Saturniidae y Sphingidae. Seguido por los humedales Paicaví, Sector Cuatro Esquinas, San Andrés y Rocuant-Andalién en los cuales se reportó una mayor abundancia de la familia Noctuidae (Cuadro N³).

\section{Cuadro $\mathrm{N}^{\circ} 3$}

Composición taxonómica, riqueza, diversidad específica y abundancia de macrolepidópteros asociados a humedales palustres del Gran Concepción

\begin{tabular}{|c|c|c|c|c|c|c|c|}
\hline Familias & Especies & L.V. & P. & S.A. & L. & C.E. & R.A \\
\hline \multirow[t]{4}{*}{ Saturniidae } & Ormiscodes sociales (Ureta) & 1 & 0 & 0 & 0 & 0 & 0 \\
\hline & Ormiscodes amphinome (Fabricius) & 1 & 0 & 0 & 0 & 0 & 0 \\
\hline & Cercophana venusta (Walker) & 1 & 0 & 0 & 0 & 0 & 0 \\
\hline & Cinommata bistrigata (Butler) & 1 & 0 & 0 & 0 & 0 & 0 \\
\hline \multirow[t]{20}{*}{ Geometridae } & Chloroclydon rinodaria (Felder \& Rogenhofer) & 3 & 0 & 0 & 0 & 0 & 0 \\
\hline & Cidaria plemirata (Felder \& Rogenhofer) & 6 & 4 & 2 & 2 & 1 & 2 \\
\hline & Euphitecia nahuelbuta Rindge & 0 & 0 & 0 & 13 & 0 & 0 \\
\hline & Ennada flavaria (Blandchard) & 0 & 1 & 0 & 1 & 0 & 0 \\
\hline & Hasodima bartleti Parra & 2 & 1 & 2 & 12 & 0 & 0 \\
\hline & Hemiloxia maditata (Felder \& Rogenhofer) & 3 & 0 & 0 & 0 & 0 & 0 \\
\hline & Hoplosauris chausi (Warren) & 2 & 0 & 0 & 0 & 0 & 0 \\
\hline & Hoplosauris indistincta (Mabille) & 1 & 0 & 0 & 0 & 0 & 0 \\
\hline & Leucolithodes paulina (Ureta) & 1 & 0 & 0 & 0 & 0 & 0 \\
\hline & Mallomus chilenaria (Felder \& Rogenhofer) & 2 & 0 & 0 & 0 & 0 & 0 \\
\hline & Perizoma pastoralis (Butler) & 1 & 0 & 0 & 0 & 0 & 0 \\
\hline & Perusia flava (Butler) & 0 & 0 & 1 & 0 & 0 & 0 \\
\hline & Perusia inusta (Felder \& Rogenhofer) & 0 & 0 & 1 & 0 & 0 & 0 \\
\hline & Perusia praecisaria (Herrich-Schäffer) & 0 & 1 & 1 & 0 & 0 & 0 \\
\hline & Psilaspilates signistriata Butler & 1 & 0 & 0 & 0 & 0 & 0 \\
\hline & Proteopharmacis valdiviata (Butler) & 0 & 0 & 0 & 0 & 0 & 1 \\
\hline & Psilaspilates venata Parra & 0 & 0 & 0 & 6 & 0 & 0 \\
\hline & Sp1nn (L.V) & 3 & 0 & 0 & 0 & 0 & 0 \\
\hline & Syncirsodes primata (Walker) & 2 & 0 & 1 & 0 & 1 & 0 \\
\hline & Xanthorhoe chiloena (Butler) & 12 & 0 & 0 & 0 & 0 & 0 \\
\hline \multirow[t]{6}{*}{ Noctuidae } & Agrotis bilitura (Guenée) & 5 & 11 & 7 & 1 & 0 & 0 \\
\hline & Agrotis hispidula (Guenée) & 4 & 10 & 10 & 0 & 0 & 0 \\
\hline & Agrotis edmidsoni (Butler) & 0 & 0 & 0 & 1 & 0 & 0 \\
\hline & Agrotis lutecens (Blanchard) & 12 & 12 & 11 & 0 & 0 & 0 \\
\hline & Elaphria bucephalina (Mabille) & 2 & 0 & 0 & 0 & 0 & 0 \\
\hline & Agrotis malefida (Guenée) & 6 & 15 & 11 & 0 & 0 & 0 \\
\hline
\end{tabular}




\begin{tabular}{|c|c|c|c|c|c|c|c|}
\hline \multirow[t]{8}{*}{ Familias } & Especies & L.V. & P. & S.A. & $\mathrm{L}$. & C.E. & R.A \\
\hline & Syngrapha gammoides (Blanchard) & 0 & 0 & 0 & 1 & 0 & 0 \\
\hline & Peridroma ambrosioides (Walker) & 1 & 1 & 30 & 0 & 0 & 0 \\
\hline & Peridroma clerica Butler & 4 & 0 & 1 & 0 & 0 & 0 \\
\hline & Faronta albilinea (Hübner) & 4 & 0 & 0 & 13 & 1 & 67 \\
\hline & Scania aspersa (Blanchard) & 8 & 0 & 0 & 3 & 0 & 2 \\
\hline & Chabuata carneado (Guering) & 0 & 1 & 0 & 0 & 0 & 0 \\
\hline & Scriptania cucutrides (Philippi) & 0 & 0 & 1 & 0 & 0 & 0 \\
\hline & Chitecomodia valdiviana (Angulo \& Olivares) & 1 & 0 & 0 & 0 & 0 & 0 \\
\hline & Pseudaletia punctulata (Blanchard) & 33 & 19 & 48 & 3 & 1 & 13 \\
\hline & Pseudoleucania aspersa (Butler) & 0 & 0 & 0 & 0 & 1 & 0 \\
\hline & Peridroma saucia (Hübner) & 11 & 17 & 23 & 1 & 0 & 0 \\
\hline & Pseudaleucania ferruginecens (Blanchard) & 7 & 6 & 0 & 1 & 0 & 0 \\
\hline & Heliothis zea (Boddie) & 1 & 0 & 0 & 1 & 0 & 0 \\
\hline & Pseudoaletia impucta (Guenée) & 9 & 27 & 38 & 1 & 1 & 12 \\
\hline & Zale lunata (Drury) & 3 & 0 & 0 & 0 & 0 & 0 \\
\hline Sphingidae & Hyles annei (Guerin) & 1 & 0 & 0 & 0 & 0 & 0 \\
\hline \multirow[t]{3}{*}{ Hepialidae } & Dalaca sp (Walker) & 2 & 0 & 0 & 0 & 0 & 0 \\
\hline & Callipielus vulgaris (Schmidt \& Robinson) & 0 & 1 & 0 & 0 & 0 & 0 \\
\hline & Dalaca pallens (Blanchard) & 1 & 0 & 2 & 0 & 0 & 0 \\
\hline Licanidae & Macromphalia ancilla (Philippi) & 0 & 1 & 0 & 0 & 0 & 0 \\
\hline Arctidae & Chilesia rudis (Butler) & 0 & 5 & 3 & 4 & 2 & 0 \\
\hline \multirow[t]{9}{*}{ Crambidae } & Sp2 & 2 & 7 & 1 & 3 & 2 & 0 \\
\hline & Sp3 & 1 & 0 & 0 & 0 & 2 & 3 \\
\hline & Sp4 & 2 & 0 & 0 & 0 & 1 & 0 \\
\hline & Sp5 & 2 & 0 & 0 & 8 & 1 & 3 \\
\hline & Sp6 & 0 & 8 & 0 & 4 & 0 & 0 \\
\hline & Sp7 & 1 & 0 & 0 & 1 & 0 & 0 \\
\hline & Sp8 & 1 & 0 & 0 & 0 & 0 & 0 \\
\hline & Nomophila sp9 & 1 & 0 & 0 & 0 & 0 & 0 \\
\hline & Sp10 & 17 & 0 & 0 & 0 & 0 & 0 \\
\hline
\end{tabular}

Nota: L.V.: Laguna Verde; P.: Paicaví; S.A.: San Andrés; R.A.: Rocuant Andalién; L.:Lenga; C.E.: Cuatro Esquinas. Fuente: Elaboración propia.

\section{Naturalidad}

Los resultados del indicador propuesto permitieron inferir el estado de preservación de la naturalidad en cada humedal. En este sentido los humedales que presentaron los valores más cercanos a 1 correspondieron a Laguna Verde $(0,67)$, Lenga $(0,47)$ y RocuantAndalién $(0,36)$; estos tres sistemas presentaron valores superior a la media $(0,29)$ de naturalidad de los humedales analizados. Finalmente los humedales Paicaví $(0,07)$, 
San Andrés $(0,04)$ y Cuatro Esquinas $(0,18)$ calificaron muy por debajo de la media, coincidiendo con una matriz muy urbanizada (Cuadro $\mathrm{N}^{\circ} 4$ ).

\section{Cuadro $\mathrm{N}^{\circ} 4$}

Variables dependientes modelos

\begin{tabular}{|l|r|r|r|}
\hline Humedales & \multicolumn{1}{|c|}{$\begin{array}{c}\text { Indicador de } \\
\text { naturalidad }\end{array}$} & Riqueza de plantas & $\begin{array}{c}\text { Riqueza de } \\
\text { macrolepidópteros }\end{array}$ \\
\hline (1) Laguna Verde & 0,67 & 5 & 44 \\
(2) Lenga & 0,43 & 4 & 20 \\
(3) Cuatro Esquinas & 0,18 & 9 & 11 \\
(4) Rocuant Andalién & 0,36 & 5 & 8 \\
(5) San Andrés & 0,07 & 8 & 19 \\
(6) Paicaví & 0,04 & 12 & 19 \\
\hline
\end{tabular}

Fuente: Elaboración propia.

\section{Modelos de Urbanización}

El análisis de correlaciones entre las variables de urbanización no consideró la superficie urbanizada, debido a que está directamente contenida en el indicador de naturalidad (Cuadro $\mathrm{N}^{\circ}$ 5). El análisis de co- rrelación arrojó algunos valores altos entre algunas de las variables explicativas, es el caso del porcentaje de superficie urbanizable y el NDVI; y entre el número de usos y la densidad de viviendas, entre las cuales existió correlaciones cercanas a la unidad (Cuadro $\left.\mathrm{N}^{\circ} 6\right)$.

Cuadro $\mathrm{N}^{\circ} 5$

Variables independientes modelos

\begin{tabular}{|c|c|c|c|c|c|c|c|c|c|c|}
\hline Humedal & Per & $\begin{array}{l}\text { Área } \\
(\mathrm{km})\end{array}$ & $\mathrm{AM}$ & DC & S.U1 & S.U2 & DP & DV & NDVI & NU \\
\hline (1) Laguna Verde & 1912,5 & 0,10 & 4,8 & 225 & 0 & 0 & 0 & 0 & 0,4 & 9 \\
\hline (2) Lenga & 14783 & 4,80 & 19,8 & 1.010 & 5,15 & 1,02 & 0 & 0 & 0,1 & 10 \\
\hline $\begin{array}{l}\text { (3) Sector } \\
\text { Cuatro Esquinas }\end{array}$ & 3127,4 & 0,24 & 6,3 & 226 & 3,90 & 0,24 & $1.238,4$ & 0,09 & 0,2 & 6 \\
\hline (4) Rocuant Andalién & 22284,5 & 11,83 & 35,8 & 841 & 31,97 & 11,43 & $1.229,5$ & 0,11 & $-0,1$ & 8 \\
\hline (5) San Andrés & 4394,3 & 0,45 & 6,6 & 405 & 8,60 & 0,57 & $4.057,0$ & 0,18 & 0,2 & 4 \\
\hline (6) Paicaví & 4681,0 & 0,25 & 7,0 & 677 & 1,91 & 0,13 & $7.136,1$ & 0,14 & 0,2 & 5 \\
\hline
\end{tabular}

Nota: Per.: Perímetro (m); DC.: Distancia a carreteras (m); S.U1.: Superficie urbanizada (\%); S.U2.: Superficie urbanizable (\%); DP.: Densidad de población (habitantes $/ \mathrm{km}^{2}$ ); AM.: Área matriz 1 km; DV.: Densidad de viviendas (viviendas $/ \mathrm{km}^{2}$ ); NU.: Número de usos.

Fuente: Elaboración propia. 
Cuadro $\mathrm{N}^{\circ} 6$

Matriz de correlaciones

\begin{tabular}{|l|r|r|r|r|r|r|}
\cline { 2 - 7 } \multicolumn{1}{c|}{} & \multicolumn{1}{c|}{ DC } & \multicolumn{1}{c|}{ S.U2 } & \multicolumn{1}{c|}{ DP } & \multicolumn{1}{c|}{ DV } & \multicolumn{1}{c|}{ NDVI } & \multicolumn{1}{c|}{ NU } \\
\hline DC & 1,000 & 0,446 & 0,018 & $-0,105$ &,- 761 & 0,391 \\
S.U2 & 0,446 & 1,000 & $-0,149$ & 0,269 &,- 840 & 0,099 \\
DP & 0,018 & $-0,149$ & 1,000 & 0,771 &, 046 & $-0,797$ \\
DV & $-0,105$ & 0,269 & 0,771 & 1,000 &,- 194 & $-0,920$ \\
NDVI & $-0,761$ & $-0,840$ & 0,046 & $-0,194$ & 1,000 & $-0,152$ \\
NU & 0,391 & 0,099 & $-0,797$ & $-0,920$ &,- 152 & 1,000 \\
\hline
\end{tabular}

Fuente: Elaboración propia.

Ante la redundancia de información se justificó un análisis de componentes principales para la extracción de las variables más significativas y aplicables a los modelos de urbanización. Considerando que el componente 1 explicó el $50 \%$, el 2 el 36,5\% y el 3 el 10,3\%, juntos explicaron una varianza acumulada del $97,7 \%$ de los datos. Por tanto, el mejor modelo explicativo en relación a in- dicadores de biodiversidad presentados en el Cuadro $\mathrm{N}^{\circ} 4$, fue el que retuvo tres variables: porcentaje de superficie urbanizable $(0,98)$, la densidad de viviendas $(0,95)$ y la distancia a carreteras $(0,89)$ (Cuadro $N^{\circ} 7$ ). Estas tres variables identificadas se utilizaron en los modelos 1, 2 y 3 como independientes para los seis humedales en estudio.

Cuadro $\mathrm{N}^{\circ} 7$

Matriz de componentes rotados

\begin{tabular}{|l|r|r|r|}
\cline { 2 - 4 } \multicolumn{1}{c|}{} & \multicolumn{3}{c|}{ Componente } \\
\cline { 2 - 4 } \multicolumn{1}{c|}{} & 1 & 2 & 3 \\
\hline DC & 0,427 & $-0,105$ & 0,890 \\
S.U2 & 0,988 & 0,046 & 0,036 \\
DV & $-0,227$ & 0,916 & 0,258 \\
NDVI & 0,237 & 0,957 & $-0,133$ \\
NU & $-0,861$ & $-0,059$ & $-0,455$ \\
& 0,117 & $-0,945$ & 0,279 \\
\hline
\end{tabular}

Fuente: Elaboración propia.

El modelo 1 incluyó como variable dependiente la riqueza de plantas y como independientes las mencionadas anteriormente. El modelo presentó un ajuste global de un $R^{2}=0,57$, indicando que este fue capaz de explicar razonablemente cómo la riqueza de plantas se vio influenciada por las variables de urbanización. Sin embargo, un $43 \%$ de la varianza no fue explicado (Cuadro $N^{\circ} 8$ ). De acuerdo a los coeficientes estandarizados, la densidad de viviendas influyó en mayor me- dida en comparación a la distancia a carreteras y el porcentaje de superficie urbanizable, las tres variables tuvieron influencias positivas; por tanto un aumento en densidad de viviendas incrementaría la riqueza de plantas en 0,75 , mientras que la distancia a carreteras en 0,13 , esto puede ser relacionado con la existencia de un mayor número de especies introducidas en las zonas más urbanizadas (Cuadro $\mathrm{N}^{\circ} 8$ ). 
Cuadro $\mathrm{N}^{\circ} 8$

Modelo 1. Riqueza de plantas

\begin{tabular}{|l|r|c|c|r|r|}
\hline \multirow{2}{*}{ Modelo } & \multicolumn{2}{|c|}{ Coeficientes tipificados } & \multirow{2}{*}{$\mathrm{t}$} & \multirow{2}{*}{ Sig. } & \multicolumn{2}{|c|}{ Intervalo de confianza de 95,0\% para B } \\
\cline { 2 - 2 } & & & & \multicolumn{1}{|c|}{ Límite inferior } & \multicolumn{1}{c|}{ Límite superior } \\
\hline (Constante) & & 0,613 & 0,602 & $-15,198$ & 20,252 \\
S.U2 & 0,020 & 0,037 & 0,974 & $-0,731$ & 0,744 \\
DC & 0,139 & 0,261 & 0,819 & $-0,024$ & 0,027 \\
DV & 0,751 & 1,511 & 0,270 & $-68,575$ & 142,791 \\
\hline
\end{tabular}

$R^{2}=0.57, \sigma=3,793 n=6$. Fuente: Elaboración propia.

El modelo 2 consideró como variable dependiente la riqueza de macrolepidópteros. Presentó un ajuste global de un $\mathrm{R}^{2}=0,76$, indicando que fue capaz de explicar razonablemente la influenciada de las variables de urbanización en la riqueza de macrolepidópteros. Sin embargo, un $24 \%$ de la varianza no fue explicado (Cuadro $N^{\circ}$ 9). Según los coeficientes estandarizados, la distancia a las carreteras influyó más que la densidad de viviendas y el porcentaje de superficie urbanizable en la variabilidad de la riqueza de macrolepidópteros. Las tres variables tuvieron una influencia negativa, por tanto a medida que disminuye la distancia de las carreteras o es más corta, la riqueza de macrolepidópteros decrecerá en 0,69, la densidad de viviendas también disminuyó la riqueza de macrolepidópteros en 0,38 (Cuadro № 9).

Cuadro $\mathrm{N}^{\circ} 9$

Modelo 2. Riqueza de macrolepidoteros

\begin{tabular}{|l|r|r|r|r|r|}
\hline \multirow{2}{*}{ Modelo } & $\begin{array}{r}\text { Coeficientes tipifi- } \\
\text { cados }\end{array}$ & \multirow{2}{*}{$\mathrm{t}$} & \multirow{2}{*}{ Sig. } & \multicolumn{2}{|c|}{ Intervalo de confianza de 95,0\% para B } \\
\cline { 2 - 2 } \cline { 5 - 5 } (Constante) & Beta & & & Límite inferior & \multicolumn{1}{c|}{ Límite superior } \\
\cline { 2 - 4 } S.U2 & $-0,185$ & $-0,452$ & 0,696 & $-2,599$ & 87,973 \\
DC & $-0,697$ & $-1,762$ & 0,220 & $-2,083$ & 1,687 \\
DV & $-0,386$ & $-1,050$ & 0,404 & $-0,092$ & 0,039 \\
& & & $-335,900$ & 204,106 \\
\hline
\end{tabular}

$R^{2}=0.76, \sigma=9,689 n=6$. Fuente: Elaboración propia.

El modelo 3 consideró como variable dependiente el indicador de naturalidad, presentó un ajuste global de un $\mathrm{R}^{2}=0,88$, indicando que es capaz de explicar considerablemente como la naturalidad de la matriz cambiaría por efecto de las variables de urbanización. Sin embargo, el $12 \%$ de la varianza estaría siendo explicado por otras variables predictoras (Cuadro $N^{\circ} 10$ ). De acuerdo a los coeficientes estandarizados el porcentaje 
de superficie urbanizable influyó de forma positiva en el indicador de naturalidad, es decir aumenta en 0,4 a medida que es mayor la superficie urbanizable, básicamente porque se mantienen las coberturas de suelo y aún no se construye, en cambio la distancia a carreteras aunque disminuya decrecerá la naturalidad. Un efecto similar se observa en la densidad de viviendas, a medida que sea menor igualmente disminuirá la probabilidad de mantener o aumentar la naturalidad. (Cuadro $N^{\circ} 10$ ).

Cuadro $\mathrm{N}^{\circ} 10$

Modelo 3. Índice de Naturalidad

\begin{tabular}{|c|c|c|c|c|c|}
\hline \multirow{2}{*}{ Modelo } & \multirow{2}{*}{$\begin{array}{c}\begin{array}{c}\text { Coeficientes } \\
\text { tipificados }\end{array} \\
\text { Beta }\end{array}$} & \multirow{2}{*}{$\mathrm{t}$} & \multirow{2}{*}{ Sig. } & \multicolumn{2}{|c|}{ Intervalo de confianza de $95,0 \%$ para B } \\
\hline & & & & Límite inferior & Límite superior \\
\hline (Constante) & & 4,519 & 0,046 & 0,030 & 1,225 \\
\hline S.U2 & 0,411 & 1,453 & 0,283 & $-0,016$ & 0,033 \\
\hline DC & $-0,297$ & $-1,085$ & 0,391 & $-0,001$ & 0,001 \\
\hline DV & $-1,014$ & $-3,982$ & 0,058 & $-6,859$ & 0,265 \\
\hline
\end{tabular}

$R^{2}=0.88, \sigma=0,127 n=6$. Fuente: Elaboración propia.

\section{Discusión y conclusión}

Concepción metropolitano está situado en un territorio costero de urbanización dispersa, muy condicionado por factores geográficos físicos como los cuerpos de agua y el relieve. Sin embargo la expansión de esta ciudad ha ido en desmedro de lagunas, humedales y vegetación nativa (Vidal \& Romero, 2010; Rojas et al., 2013a), en primer lugar perdiendo áreas de alta calidad ambiental de los humedales de Lenga, Rocuant-Andalién y Los Batros, por vegetación dispersa, áreas sin vegetación y urbanización (Smith \& Romero, 2009) y en segundo la diversidad de especies de los humedales palustres (Villagrán et al., 2006; Sepúlveda et al., 2012).

Un primer efecto de la urbanización se observa sobre el indicador de naturalidad (IN). Los humedales con menor grado de perturbación o las naturalidades más altas fueron Laguna Verde y Lenga. En el humedal Laguna Verde influye positivamente su ubicación al interior de la reserva natural Hualpén, rodeado por una matriz más natural, dominada por bosque nativo, mientras que el humedal Lenga aunque se encuentra localizado muy cercano a la reserva, tiene una perturbación mayor debido a la urbanización del entorno, la cual corresponde a una caleta de pescadores de baja densidad según lo observado en terreno, sin embargo, la temporalidad de los datos censales no permite ver en profundidad el efecto de la densidad de población y viviendas en este humedal.

Los humedales con un entorno menos urbanizado como Laguna Verde y Lenga presentan una mayor riqueza de macrolepidópteros, principalmente por la escasa presencia de flora introducida, factor clave que determina las diferencias entre estos ecosistemas. Laguna Verde destaca por la presencia de plantas nativas en comparación a los humedales palustres de Paicaví, San Andrés y Cuatro Esquinas, Ios cuales presentan mayor heterogeneidad vegetacional, siendo 
las plantas halófitas las dominantes y una menor diversidad de insectos, tendencia evidenciada desde el año 2006 (Villagrán et al., 2006; Sepúlveda et al., 2012). Los resultados de alta y baja naturalidad son coincidentes con Centro EULA (2011), quienes identifican los sistemas Laguna Verde en una calidad muy buena, y a Paicaví como muy mala. Sin embargo, difieren en los humedales Lenga (calidad mala) y San Andrés (calidad media), esto puede ser explicado por la metodología aplicada, la que utiliza el Índice del Estado de Conservación de Ecosistemas Lenticos Someros (ECELS).

Respecto al humedal Rocuant-Andalién presenta una menor riqueza de macrolepidópteros y mayor presencia de flora introducida, características que pueden ser vinculadas a sus condiciones históricas de contaminación, que durante la década de 1980 lo transforman en un sistema eutroficado. Debido al vertimiento directo de efluentes de la industria pesquera aledaña y de aguas servidas, los sedimentos se cubrieron de una gruesa capa de fangos anóxicos, lo que fue asociado con una pérdida total de su flora y fauna característica (Rudolph y Ahumada, 1987).

Los modelos permitieron observar que las variables urbanas más influyentes sobre la biodiversidad de los humedales analizados, fueron el porcentaje de superficie urbanizable, la distancia a carreteras y la densidad de viviendas, afectando directamente a todos los humedales. El estudio demuestra que los humedales con mayor número de especies nativas y mayor naturalidad están más lejos de las cabeceras urbanas. La riqueza de plantas tiene una mayor probabilidad de variación positiva por efecto del aumento de la densidad de las viviendas, mientras que la riqueza de macrolepidópteros se reduce por la disminución de la distancia a carreteras o acercamiento a estas. Aunque el efecto de esta distancia es diferenciado, ya que el humedal Laguna Verde se encuentra muy cercano a una vía de transporte dentro de la Reserva, pero su uso se debe a la recreación y al turismo, en cambio en el humedal Rocuant-Andalién la distancia media es mayor, pero a una vía de transporte de mayor flujo como la Ruta Interportuaria Talcahuano-Penco (http://www. interportuaria.cl), además como se puede ob- servar en la Figura $N^{0}$ 1, su construcción ha fragmentado el humedal.

La variable superficie urbanizable que incorpora el proceso de urbanización futura proporcionó evidencias interesantes de observar, por ejemplo ha resultado más significativa que el valor de NDVI y el número de usos. De acuerdo a la urbanización planificada, el humedal Rocuant-Andalién sería el más perjudicado, ya que además de lo urbanizado desde el año 1975 (Smith y Romero, 2009) se espera que un $11 \%$ más de su superficie se construya, aunque es un escenario potencial, ya que el Plan Regulador Metropolitano de Concepción, vigente desde el año 2003, se encuentra en proceso de modificación e incluye entre uno de sus ejes estratégicos acciones sobre el entorno natural y construido, que podrían modificar la planificación del suelo del humedal (http://www.prmconcepcion.cl).

Es necesario recalcar lo preocupante que resultan estos resultados en el humedal Rocuant-Andalién, siendo un ecosistema relevante para la migración de la avifauna asociada (Centro EULA, 2011) y para el drenaje de aguas Iluvia, función importantísima en este territorio, debido los recurrentes periodos de inundaciones fluviales, las que se han registrado en 21 ocasiones entre 1960-2010 (Rojas, 2015). Además la zona ha brindado históricamente protección ante eventos de tsunamis, como lo demuestran los depósitos de antiguos eventos (e.g. 1835) y del reciente tsunami de 2010 (Beltrán, 2010; Valdivia y Lagos, 2014). Estos servicios podrían ser eliminados o reducidos por proyectos de urbanización, por ejemplo el proyecto de plataforma logística requiere de un análisis crítico y participación ciudadana, debido a que contempla una intensificación de los usos de suelo, incluyendo un parque industrial, comercial, servicios, recreación y solo una zona destinada a protección ecológica (http:// www.plataformalogistica.cl).

Se infiere que los humedales de Concepción con una matriz menos intervenida, presentan una mayor preservación de su biodiversidad natural, lo cual permitiría conservar un mayor número de funciones ecosistémicas que humedales que son más homogéneos desde el punto de vista de las especies y 
por tanto su estructura (Loreau et al., 2001, Moreno-Mateos et al., 2012). Esto tiene relación con la provisión de servicios ecosistémicos tanto directa como indirectamente. Por ejemplo la depuración de agua (MEA, 2005), es realizada por una comunidad diversa de especies de plantas, por lo tanto una comunidad vegetacional más diversa presentaría un mejor rendimiento en dicha función. De manera directa, un humedal con mayor diversidad de plantas o mayores índices de naturalidad, generaría mayores beneficios desde el punto de vista de los servicios ecosistémicos de recreación y bienestar psicológico de la comunidad. Diversos estudios han mostrado correlaciones positivas entre el grado de naturalidad de áreas verdes urbanas con variables de bienestar humano (Irvine et al., 2010), y en algunos casos han correlacionado incluso la riqueza de plantas, positivamente con variables psicológicas del bienestar y motivación para visitar áreas verdes urbanas (Dallimer et al., 2012, Fuller et al., 2007).

Los resultados presentados ayudan a la reflexión sobre la idea de desarrollo sustentable en Concepción metropolitano. En efecto, Aliste et al. (2012) reconoce que a partir de los años 80 es cuando más se pierden superficies de humedales, sin embargo coexisten las mejores condiciones para un progreso basado en el incremento de las residencias e industrias. Entonces, si no conciliamos el progreso con la sustentabilidad es probable que los humedales urbanos solo se mantengan cuando no existan las condiciones económicas para seguir construyendo. De acuerdo a concepciones de sustentabilidad se recomiendan algunos lineamientos generales en la normativa actual, como la inclusión de los humedales urbanos en la planificación metropolitana como áreas de protección y/o biodiversidad urbana, otra alternativa es incorporarlos como reserva natural urbana dentro del reciente proyecto de Ley que crea el Servicio de Biodiversidad y Áreas Protegidas.

Desde aspectos más específicos, se recomienda evitar la formación de microbasurales en las cercanías de los humedales puesto que pueden pasar rápidamente a ser lugares de riesgo para la salud. Factores que contribuyen a esto son la intensificación del uso de sus bordes, la reducción de la biodiversidad (incluyendo depredadores naturales de orga- nismos vectores de enfermedades), y cambios en la resistencia de patógenos a métodos de control, debido al uso excesivo de pesticidas o antibióticos, entre otros (Bridgewater, 2011). Se deben reducir los rellenos, la construcción de carreteras sobre los humedales, los desagües sin tratamiento terciario, y el vertido de fertilizantes. Se requiere aumentar el conocimiento, investigación y comunicación sobre los humedales para reconocer su importancia y subsecuente valoración por parte de la población.

Los tipos de urbanización desarrollados sobre humedales ya no son ajenos a la ciudadanía, si bien antes no existía conciencia de su importancia, incluso se les consideraba pantanos sin valor ecológico (Aliste et al., 2012) hoy existe preocupación por la coexistencia de los humedales con el crecimiento urbano, así como por la construcción de carreteras sobre estos, especialmente porque su desaparición influye en el aumento de la vulnerabilidad ante riesgos (CEDEUS, 2013, Barbosa \& Villagra, 2015). Nos atrevemos a proyectar que la preservación de los humedales, así como el aumento de los espacios abiertos y verdes de los cuales los humedales son partícipes, serán una demanda ciudadana creciente.

Concepción tiene amplios desafíos y oportunidades, especialmente el cómo vivenciamos una ciudad sustentable. En otras ciudades más industrializadas como Londres se destruyeron los humedales, pero hoy están centrados en su recuperación (http://www. wwt.org.uk/). Por otro lado, cuando estos ambientes son destruidos existen alternativas de restauración, sin embargo constituyen técnicas costosas, requieren de un conocimiento detallado del ecosistema para su manejo adaptativo y que su necesidad se inserte en políticas públicas, estas últimas de lenta generación (Hackney, 2000, Kentula, 2000, Zhang \& Shao, 2013, Tenório et al., 2015). En Concepción existe la oportunidad de innovar y crear proyectos urbanos que incorporen criterios ecológicos y de arquitectura del paisaje para crear espacios de conservación y de transición que incluso pueden tener uso recreativo y educativo. Al respecto Forman (2014) señala que un modelo basado en humedales más carreteras y construcciones no tiene impactos negativos en comparación a 
un modelo basado solo en carreteras y viviendas, e incluso una ciudad en la que conviven humedales y construcción, tiene impactos positivos sobre organismos del suelo, depósitos de erosión, vegetación, vegetación acuática, biodiversidad, árboles de riveras de ríos y aves.

Finalmente consideramos que la riqueza de especies y naturalidad de los humedales urbanos constrastados con variables de urbanización, permiten evaluar la biodiversidad en relación a estos patrones, facilitando y sintetizando el estudio de estos ecosistemas, dando un soporte a la prevención de la pérdida de biodiversidad de los humedales por estos factores, para mejorar las políticas de conservación y de desarrollo urbano, relevando que el conocer la situación de los humedales urbanos y ver su estado, ya sea seminatural, natural o muy intervenido es vital para habitar ciudades más sustentables.

\section{Referencias bibliográficas}

ALISTE, E.; ALMENDRAS, A. y CONTRERAS, M. La Dinámica del territorio en la conurbación Concepción-Talcahuano: Huellas urbanas para una interpretación de las transformaciones ambientales durante la mitad del siglo XXI. Revista de Geografía Norte Grande, 2012, No 52, p. 5-18.

AGUAYO, M.; WIEGAND, T.; AZÓCAR, G.; WIEGAND, K. \& VEGA, C. Revealing the driving forces of mid-cities urban growth patterns using spatial modeling: a case study of Los Ángeles, Chile. Ecology and Society, 2007, Vol. $12, N^{\circ} 1$. Disponible en Internet: http://www. ecologyandsociety.org/vol12/iss1/art13/

AL-AHMADI, K.; SEE, L.; HEPPENSTALL, A. \& HOGG, J. Calibration of a fuzzy celular autómata model of urban dynamics in Saudi Arabia. Ecological Complexity, 2009, № 6 , p. 80-101.

AÑASCO, N. Desarrollo estacional y relaciones de competencia entre Scirpus californicus, Typha angustifolia y Phragmites communis en pantanos de Valdivia. Valdivia. Tesis de Magíster, Universidad Austral de Chile, 1981.

ADAME, M.; KAUFFMAN, J.; MEDINA, I.; GAMBOA, J.; TORRES, O.; CAA-
MAL, J.; REZA, M. \& HERRERA-SILVEIRA, J. Carbon Stocks of Tropical Coastal Wetlands within the Karstic Landscape of the Mexican Caribbean. PLoS ONE, 2013 , Vol. 8, $N^{\circ}$ 2. Disponible en Internet: DOI: 10.1371/journal.pone.0056569

ANDRADE-C, M.G. Utilización de las mariposas como bioindicadores del tipo de hábitat y su diversidad en Colombia. Revista Academia Colombiana de Ciencias, 1998, Vol. 22, No 84, p. 407-421.

BARRAGÁN, J. Medio Ambiente y Desarrollo en Áreas Litorales. Introducción a la Planificación y gestión Integradas. Cádiz: Universidad de Cádiz, Servicio de Publicaciones, 2003.

BARBOSA, O. \& MARQUET, P.A. Effects of forest fragmentation on the beetle assemblage at the relict forest of Fray Jorge, Chile. Oecologia, 2002, No 132 , p. 296-306.

BARBOSA, O. \& VILLAGRA P. SocioEcological Studies in Urban and Rural Ecosystems in Chile. In: ROZZI, R; CHAPIN, S.F.; CALLICOTT, J.B.; PICKETT, S.T.A.; POWER, M.; ARMESTO, J.J. \& MAY JR., R.H. (editors). Earth Stewardship: linking ecology and ethics in theory and praxis. Springer Verlag, 2015, p. 297-311.

BERTRÁN, C.; VARGAS, L.; PEÑA, F.; SCHLATTER, R.; TAPIA, J. y HAUENSTEIN E. Distribución de la macrofauna bentónica en el lago costero Budi, Sur de Chile. Revista de Biología Marina y Oceanografía, 2010, Vol. $45, N^{\circ} 2$, p. $235-243$.

BELTRÁN, M. Transformaciones espaciales y valoración social del humedal RocuantAndalién (Concepción, Chile): periodo de 1950 a 2011. Santiago de Chile: Universidad de Chile, 2010.

BOLUND, P. \& HUNHAMMAR, S. Ecosystem services in urban areas. Ecological ECOnomics, 1999, № 29, p. 293-301.

BORJA, A.; GALPARSORO, I.; SOLAUN, O.; MUXIKA, I.; TELLO, M.; URIARTE, A. \& VALENCIA, V. The European Water Framework Directive and the DPSIR, a methodological approach to assess the risk of failing 
to achieve good ecological status. Estuarine, Coastal and Shelf Science, 2006, № 6, p. 8496.

BRIDGEWATER, P. Water services, Urban Landscapes. In: NIEMELÄ, J.; H. BREUSTE, J.; GUNTENSPERGEN, G.; MCINTYRE, N.; ELMQVIST, T. \& JAMES, P. (editors). Urban Ecology: Pattern, Processes and Applications. Oxford: Oxford University Press, 2011, p. 219-227.

BROWN, K.S. Palaeoecology and regional patterns of evolution in neotropical forest butterflies. In: PRANCE, G.T. (editor). Biological diversification in the tropics. New York: Columbia University Press, 1982, p. 336-357.

BROWN, K.S. Diversity, disturbance, and sustainable use of Neotropical forest: insects as indicators for conservation monitoring. Journal of Insect Conservation, 1997, $\mathrm{N}^{0} 1$, 25-42.

CAMPUZANO, M.; MATEUS, D.; LEITÃO, P.; MARÍN, V.; DELGADO, L.; TIRONI, A.PIERINI, J.; SAMPAIO, A.; ALMEIDA, P. \& NEVES, P. Integrated coastal zone management in South America: A look at three contrasting systems. Ocean \& Coastal Management, 2013, $\mathrm{N}^{\circ} 72$, p. 22-35.

CASTRO, M.; BAHAMONDES, M.; SALAS H. y AZÓCAR, P. Identificación y Ubicación de Áreas de Vegas y Bofedales de las Regiones Primera y Segunda. Santiago de Chile: Facultad de Ciencias Sociales, Universidad de Chile - Dirección General de Aguas, Ministerio de Obras Públicas, 1993.

CARRASCO, P. Variaciones de la estructura de la avifauna a lo largo de un gradiente de salinidad en el humedal Tubul-Raqui, VIII Región, Chile. Concepción: Unidad de Investigación, Universidad de Concepción, 2003.

CEDEUS. Síntesis principales resultados Conversatorios Urbanos. Concepción: Centro de Desarrollo Urbano Sustentable, 2013. Disponible en Internet:

http://www.cedeus.cl/wp-content/ uploads/2014/08/Informe-Ejecutivo-Conversatorios-Urbanos-Concepcion.pdf
CENDRERO, A. \& FISCHER, D. A procedure for assessing the environment quality of coastal areas for plannings and management. Journal of Coastal Research, 1997, Vol 13, No 3, p. 732-744.

CARTER, R. Coastal Environments. London: Academic Press, 1988.

CENTRO EULA. Diagnóstico y propuesta para la conservación y uso Sustentable de los humedales lacustres y urbanos. Principales de la región del Biobío. Propuesta Metodológica para el Manejo y Gestión para Humedales de la Región del Biobío. Concepción: Centro de Ciencias Ambientales, Universidad de Concepción, 2011.

COLLINS, M.E. \& KUEHL, R.J. Organicmatter accumulation and organic soils. In: RICHARDSON, J.L. \& VEPRASKAS, M.J. (editors). Wetland soils: genesis, hydrology, landscapes and classification. Boca Raton: Lewis Publishers, 2000, p. 137-162.

COMISIÓN NACIONAL DEL MEDIO AMBIENTE (CONAMA). Evaluación del estado de Conservación de las Aves de Humedal de la Región del Bío-Bío, Chile. Santiago de Chile: CONAMA, 1999.

COMISIÓN NACIONAL DEL MEDIO AMBIENTE (CONAMA). Diagnóstico y propuesta de manejo de los humedales de la región del Maule. Santiago de Chile, CONAMA, 2000.

COMISIÓN NACIONAL DEL MEDIO AMBIENTE (CONAMA). Estrategia para la conservación y uso racional de los humedales en Chile. Santiago de Chile: CONAMA, Santiago, 2005.

COMISIÓN NACIONAL DEL MEDIO AMBIENTE CONAMA-CEA. Protección y manejo sustentable de humedales integrados a la cuenca hidrográfica. Santiago de Chile: CONAMA-CEP, 2006.

CONSTABEL, S. Ambientes sedimentarios de los estuarios Tubul y Raqui, VIII Región, Chile. Concepción: Tesis para optar al Grado de Doctor en Ciencias Ambientales, Escuela de Graduados de la Universidad de Concepción, 1993 
COWARDIN, L.M.; CARTER, V.; GOLET, F.C. \& LAROE, E.T. Classification of wetlands and deepwater habitats of the United States. Washington: U.S Department of the Interior, Fish and Wildlife Service, Northern Prairie wildlife Research Center, 1979.

CHEN, W.Y. \& JIM, C.Y. Cities, 2008, No 25, p. 298-309.

CVETKOVIC, M. \& CHOW-FRASER, P. Use of ecological indicators to assess the quality of Great Lakes coastal wetlands. Ecological Indicators, 2011, № 11, p.1609-1622.

DALLIMER, M.; IRVINE, K.N.; SKINNER, A.M.J.; DAVIES, Z.G.; ROUQUETTE, J.R.; MALTBY, L.L. \& GASTON, K.J. Biodiversity and the Feel-Good Factor: Understanding Associations between Self-Reported Human Well-being and Species Richness. BioScience, 2012, Vol. 62, No 1, p. 47-55.

DUFRENE, M. \& LEGENDRE, P. Species assemblages and indicator species: the need for a flexible asymmetrical approach. Ecological. Monographs, 1997, Vol. 67, № 3, p. 345-366.

ELLIOTT, M.; BURDON, D.; HEMINGWAY, K. \& APITZ, S. Estuarine, coastal and marine ecosystem restoration: confusing management and science - A revision of concepts. Estuarine, Coastal and Shelf Science, 2007, № 74, p. 349-366.

ESPINOZA, A. Caracterización natural del humedal de Price en Talcahuano: Una compilación de información científica para restablecer una línea base para el manejo del ecosistema. Curso-Taller " Gestión Ambiental Municipal, 1997, p. 70-102.

FORMAN, R.T.T. Urban Ecology: Science of Cities. Cambridge: Cambridge University Press, 2014.

FROST, S.W. Light traps for insect collection, survey and control. Bulletin of the Pathology Agriculture Experimental Station, 1952, No 550 .

FROST, S. W. The Pennsylvania insect light trap. Journal of Economic Entomology, 1957, № 50, p. 287-92.
FULLER, R.A.; IRVINE, K.N.; DEVINEWRIGHT, P.; WARREN, P.H.; \& GASTON, K.J. Psychological benefits of greenspace increase with biodiversity. Biology Letters, 2007, Vol. 3, № 4, p. 390-394.

GALLO M. y RODRÍGUEZ, E. Caracterización de paisajes y ecosistemas: proyecto demostrativo cuenca baja del río Paz El Salvador - Guatemala. Wetlands international, 2010, p. 1- 41.

GIBB, H. \& HOCHULLI, F. Habitat fragmentation in an urban environment: large and small fragments support different arthropod assemblages. Biological Conservation, 2002, No 106, p. 91-100.

GOMEZ-BAGGENTHUN, E. \& BARTON. D. Classifying and valuing ecosystem services for urban planning. Ecological Economics, 2013, Vol. 86, p. 235-245.

GONZÁlEZ, A. y VICTORIANO, P. Aves de los humedales costeros de la zona de Concepción y alrededores. En: SMITH-RAMÍREZ, C; ARMESTO, J. y VALDOVINOS, C. (editores). Historia, biodiversidad y ecología de los bosques costeros de Chile. Santiago de Chile: Editorial Universitaria Bosque Nativo, 2005, p. 485-497.

HACKNEY, C. Restoration of coastal habitats: expectation and reality. Ecological Engineering, 2000, $\mathrm{N}^{\circ} 15$, p. 165-170.

HAUENSTEIN, E.; GONZÁLEZ M, PEÑACORTÉS F. y MUÑOZ- PEDREROS A. Clasificación y caracterización de la flora y vegetación de los humedales de la costa de Toltén (IX Región, Chile). Gayana Botánica, 2002, Vol. 59, № 2, p. 87-100.

ILABACA, P. Evolución de la costa de Concepción: el caso de las bahías de Concepción y San Vicente. Biología Pesquera, 1989, No 18, p. 29-35.

HOFFMANN, A. Flora Silvestre de Chile. Zona Central. Santiago de Chile: Ediciones Fundación Claudio Gay, 1998.

HOLLINGSWORTH, J.P.; HARTSOCK, J.G. \& STANLEY, J.M. Electrical Insect traps for survey purposes. Washington: Agricultural 
Research Service United States, Department of Agriculture 42-3-1, 1963.

IRVINE, K.N.; FULLER, R.A.; DEVINEWRIGHT, P.; PAYNE, S.; TRATALOS, J.; WARREN, P.; LOMAS, K.J. \& GASTON, K.J. Ecological and psychological value of urban green space. In: JENKS, J. \& JONES, C. (editors). Dimensions of the Sustainable City. Amsterdam: Springer, 2010, p. 215-237.

ISLA, F.; QUEZADA, J.; MARTÍNEZ, C.; FERNÁNDEZ, A. \& JAQUE, E. The evolution of the Bío Bío delta and the coastal plains of the Arauco Gulf, Bío Bío Region: the Holocene sea-level curve of Chile. Journal of Coastal Research, 2012, No 28, p. 102-111.

JIMÉNEZ, M. Evaluación del estado de Conservación de las aves de humedal de la Región del Bío Bío. Concepción: Habilitación profesional, Facultad de Ciencias Naturales y Oceanográficas, Universidad de Concepción \& CONAMA, 1999.

KENTULA, M. Perspectives on setting success criteria for wetland Restoration. ECOlogical Engineering, 2000, №15, p. 199-209.

KOCH, E.; BARBIER,E.; SILLIMAN,B.; REED,D.; ME PERILLO, G.; HACKER, S.; GRANEK,E.; PRIMAVERA, J.; MUTHIGA, N.; POLASKY,S.; HALPERN, B.; KENNEDY,C.; KAPPEL,C. \& WOLANSKI, E. Non-linearity in ecosystem services: temporal and spatial variability in coastal protection. Frontiers in Ecology and the Environment, 2009, № 7, p. 29-37..

KREMEN, C. Biological inventory using target taxa. A case study of butterflies of Madagascar. Ecological Application, 1994,Vol .4, $\mathrm{N}^{\circ} 3$, p. 407-422.

LOREAU, M.; NAEEM, S.; INCHAUSTI, P.; BENGSTSSON, J.; GRIME, J.P.; HECTOR, A, HOOPER, D.U.; HUSTON, M.A.; RAFFAELLI, D.; SCHMID, B. \& WARDLE, D.A. Biodiversity and Ecosystem Functioning: Current Knowledge and Future Challenges. Science, 2001, Vol. 294, No 5543, p. 804-808.

NIEMELA, J.; SAARELA, S.; SODERMAN, T.; KOPPEROINEN, L.; YLI-PELKONEN, V.; VAARE, S. \& KOTZE, D. Using the ecosystem services approach for better planning and conservation of urban green spaces: a Finland case study. Biodiversity Conservation, 2010, No 19, p. 3225-3243.

MARQUES, A.; RAMOS, T.; CAEIRO, S. \& COSTA, M. Adaptive-participative sustainability indicators in marine protected areas: Design and communication. Ocean \& Coastal Management, 2013, № 72, p. 36-45.

MACARTHUR, R.H. \& WILSON, E.O. The theory of island biogeography. Princeton University Press, Princeton, 1967.

MATTHEI, O. Manual de las malezas que crecen en Chile. Santiago de Chile: Alfabeta Impresores, 1995.

MCLACHLAN, A.; DEFEO, O.; JARAMILLO, E. \& SHORT, D. Sandy beach conservation and recreation: Guidelines for optimising management strategies for multi-purpose use. Ocean \& Coastal Management, 2013, № 71, p. 256-268.

MILLENNIUM ECOSYSTEM ASSESSMENT (MEA). Millennium Ecosystem Assessment Synthesis Report. Washington: Island Press, D.C, 2005.

MITSCH, W.J. \& GOSSELINK, J.G. Wetlands. New York: John Wiley \& Sons, Inc, 2000.

MINISTERIO DE MEDIO AMBIENTE (MMA)- CENTRO DE ECOLOGÍA APLICADA (CEA). Diseño del inventario nacional de humedales y el seguimiento ambiental. Ministerio de Medio Ambiente. Santiago de Chile, MMA-CEA, 2011.

MORENO-MATEOS, D.; POWER, M.E.; COMÍN, F. \& YOCKTENG, R. Structural and Functional Loss in Restored Wetland Ecosystems. PLos Biology, 2012, Vol. 10, № 1. Disponible en Internet: e1001247. doi:10.1371/ journal.pbio. 1001247.

MORENO-MAY, G.J.; CERÓN-BRETÓN, J.G.; CERÓN-BRETÓN, R.M.; GUERRA-SANTOS, J.J.; AMADOR DEL ÁNGEL, L.E. y ENDAÑÚ-HUERTA, E. Estimación del potencial de captura de carbono en suelos de manglar de isla del Carmen. U. Tecnociencia, 2010, 4, $N^{\circ} 1$, p. $23-39$. 
MUÑIZ, I \& GALINDO, M. Urban Form and the Ecological Footprint of Commuting. The Case of Barcelona. Ecological Economics, 2005, No 55, p. 499-514.

PEÑA-CORTÉS F.; GUTIÉRREZ P.; REBOLLEDO G.; ESCALONA M.; HAUENSTEIN, E.; BERTRÁN, C. \& TAPIA, J. Determinación del nivel de antropización de humedales como criterio para la planificación ecológica de la cuenca del lago Budi, IX Región de la Araucanía, Chile. Revista de Geografía Norte Grande, 2006, № 36, p. 75-91.

PARRA, O.; DELLAROSA, V.; CONEJEROS, M.; VALDEBENITO, S.; CASTILLO, R.; HIDALGO, A.; ARIAS, P.; DÍAZ, S.; GAETE, B.; CAMPOS, H.; STEFFES, W. y UGARTE, E. Estudio de la eutroficación de la laguna Grande de San Pedro y la laguna Tres Pascualas. Concepción: Universidad de Concepción, 1989.

PARRA, O.; DELLA CROSE, N. y VALDOVINOS, C. Elementos de limnología teórica y aplicada. Roma: Microart's Edizioni, 2004.

PAUCHARD, A.; AGUAYO, M.; PEÑA, E. \& URRUTIA, R. Multiple effects of urbanization on the biodiversity of developing countries: The case of a fast-growing metropolitan area (Concepción, Chile). Biological Conservation, 2005, No 127 , p. 272-281.

PLATA, W.; GÓMEZ, M. y BOSQUE SENDRA, J. Desarrollo de modelos de crecimiento urbano óptimo para la comunidad aplicando métodos de evaluación multicriterio y sistemas de información geográfica. Revista Internacional de Ciencia y Tecnología de la Información Geográfica Geofocus, 2010, № 10, p. 103-134.

PINO, J., VILÁ, M.; ALVÁREZ, N.; SEGUÍ, J. \& GUERRERO, C. Niche breadth rather than reproductive traits explains the response of wetland monocotyledons to land cover change. Applied Vegetation Science, 2009, No 12 , p. 9-21.

QUEZADA, A.; OYARZO, E.H. y RUIZ V.H. Distribución de avifauna en los distintos hábitats del Estuario Andalién, Bahía de Concepción, Concepción, Chile. Studies on Neotropical Fauna and Enviroment, 1986, N ${ }^{\circ}$ 21, p. 197-206.
QUINTANA, V. Caracterización florística y faunística de un humedal costero de la VIII región, el caso del Estero Lenga. En: Planificación y gestión de la zona costera. Análisis de un caso: Lenga. Concepción: Centro EULAChile, Universidad de Concepción. Serie: Propuesta de Ordenamiento $N^{\circ}$ 8, 1993, p. 41-56.

RAMÍREZ, C.; SAN MARTÍN, C. y RUBILAR, H. Una propuesta para la clasificación de humedales chilenos. Revista Geográfica de Valparaíso, 2002, No 32-33, p. 265-273.

RIEDEMANN, P. y ALDUNATE, G. Flora Nativa de Valor Ornamental. Identificación y Propagación. Chile, Zona Sur. Santiago de Chile: Editorial Andrés Bello, 2003.

RIEDEMANN, P. y ALDUNATE, G. Flora Nativa de Valor Ornamental. Identificación y Propagación. Chile, Zona Centro. Santiago de Chile: Editorial Andrés Bello, 2004.

RODRIGUES, R.; MASCARENHAS, A. \& JAGTAP, T. An evaluation of flora from coastal sand dunes of India: Rationale for conservation and management. Ocean \& Coastal Management, 2011, № 54, p. 181-188.

ROJAS, C.; PINO, J.; BASNOU, C. \& VIVANCO, M. Assessing land use and cover changes in relation to geographic factors and urban planning in the Metropolitan Area of Concepción (Chile). Implications for biodiversity conservation. Applied Geography, 2013a, No 39, p. 93-103.

ROJAS, C.; PINO, J. \& JAQUE, E. Strategic Environmental Assessment in Latin America: a methodological proposal for Urban Planning in the Metropolitan Area of Concepción (Chile). Land Use Policy, 2013b, No 30, p. 519-527.

ROJAS, C.; PLATA, W.; VALDEBENITO P.; MUÑIZ, I. y DE LA FUENTE, H. La Dinámica de expansión urbana del Área Metropolitana de Concepción. En: WILLIAMS, J.; HIDALGO, R.; BRAND, P. y PÉREZ, L. (editores). Metropolizaciones Colombia -Chile: Experiencias de Bogotá, Medellín, Santiago y Concepción. Bogotá: Línea Editorial 1 Investigaciones, Universidad Nacional de Colombia, 2014, p. 39-56. 
ROJAS, O. Cambios Ambientales y Dinamica de Inundaciones Fluviales en una Cuenca Costera del Centro Sur de Chile. Concepción: Tesis doctoral, Universidad de Concepción, 2015.

RUDOLPH, A. y AHUMADA, R. Intercambio de nutrientes entre una marisma con una fuerte carga de contaminantes orgánicos y las aguas adyacentes. Boletín Sociedad Biología, 1987, No 58, p. 151-169.

SAN MARTÍN, C.; CONTRERAS, D.; SAN MARTÍN J. y RAMÍREZ C. Vegetación de las marismas del centro-sur de Chile. Revista Chilena de Historia Natural, 1992, No 65, p. 327-342.

SAN MARTÍN, C.; SUBIABRE, M. y RAMÍREZ, C. Estudio florístico y vegetacional de una gradiente latitudinal en marismas del centro-sur de Chile. Ciencia e Investigación Agraría, 2006, Vol. 33, Nº 1, p. 37-45.

SEKOVSKI, I.; NEWTON, A. \& DENISSON, W. Megacities in the coastal zone: Using a driver - pressure - state-impact-response framework to adress complex environmental problems. Estuarine, Coastal and Shelf Science, 2012, Vol. 96, p. 48-59.

SEINGIERA, G.; ESPEJEL, I.; FERMÁN-ALMADAA, J.L.; MONTAÑO-MOCTEZUMAC, G.; AZUZ-ADEATHD, I. \& ARAMBUROVIZCARRAE, G. Mexico's coasts: Half-way to sustainability. Ocean \& Coastal Management, 2011, No 54, p. 123-128.

SEINGIER, G. ESPEJEL, I. FERMÁN-ALMADA, J. MONTAÑO-MOCTEZUMA, G. AZUZADEATH, I. \& ARAMBURO-VIZCARRA, G. Mexico's coasts: Half-way to sustainability. Ocean \& Coastal Management, 2011, No 54, p. 123-128.

SEPÚLVEDA, E.; PARRA, L.; BENÍTEZ, H. y ROJAS, C. Estados de naturalidad y heterogeneidad vegetacional de humedales palustres y su efecto sobre la diversidad de Macrolepidoptera (Insecta: Lepidoptera). SHILAP Revista Lepidóptera, 2012, Vol. 40, No 158, p. $155-170$.

SHERMAN, D. \& BAUER, B. Coastal geomorphology through the looking glass. Geomorphology, 1993, № 7, p. 225-249.
SMITH, P. y ROMERO, H. Efectos del crecimiento urbano del Área Metropolitana de Concepción sobre los humedales de RocuantAndalién, Los Batros y Lenga. Revista de Geografía Norte Grande, 2009, No 43, p. 81-93.

STUARDO, J. y VALDOVINOS, C. Estuarios y lagunas costeras: Ecosistemas Importantes de Chile Central. Ambiente y Desarrollo, 1989, No 5, p. 107-115.

STUARDO, J.; VALDOVINOS, C.; FIGUEROA, R. y OCHIPINTI, A. Los ambientes costeros del Golfo de Arauco y área adyacente. Concepción: Serie Monografías Científicas, Centro EULA (Chile), 1993, p. 31-43.

SUMMERVILLE, K.O.; RITTER, L. \& THOMAS, C. Forest moth taxa indicators of lepidopteran richness and habitat disturbance: a preliminary assessment. Biological Conservation, 2004, No 116, p. 9-18.

TENÓRIO, G.; SOUZA-FILHO, P.W.; RAMOS, E. \& ALVES, P. Mangrove shrimp farm mapping and productivity on the Brazilian Amazon coast: Environmental and economic reasons for coastal Conservation. Ocean \& Coastal Management, 2015, №104, p. 65-77.

UNITED NATIONS (UN). World Urbanization Prospects: The 2014 Revision, Highlights (ST/ESA/SER.A/352). Department of EConomic and Social Affairs, Population Division. 2014. Disponible en Internet: http:// esa.un.org/unpd/wup/Highlights/WUP2014Highlights.pdf

VALDIVIA, D. y LAGOS, M. Reconocimiento y caracterización de depósitos del tsunami de 2010 en la bahía de Concepción, Chile. En: Congreso de la Sociedad Chilena de Ciencias Geográficas. Coyhaique, noviembre de 2014 .

VALDOVINOS, C. Caracterización Ambiental del Estuario Lenga, VIII Región, Chile. Planificación y Gestión de la Zona Costera. Análisis de un caso: Lenga Concepción. Concepción: Centro EULA, Universidad de Concepción, 1993.

VALDOVINOS, C. Humedales dulceacuícolas y biodiversidad. En: ROJAS, J.; AZÓCAR, G.; MUÑOZ, M.D.; VEGA, C.; KINDLER, A. y KABISCH, S. Atlas social y 
ambiental del área metropolitana de Concepción, Región del Biobío, Chile: Transformaciones sociodemográficas y ambientales 19922002. Concepción: Editorial Universidad de Concepción, 2006, p. 104-124.

VÁSQUEZ, D. Metodología para la delimitación y zonificación de humedales costeros de tipo mediterráneo. Revista Geográfica del Sur, 2013, Vol. 4, № 6, p. 13-32.

VILLAGRAN-MELLA, R.; AGUAYO, M.; PARRA, L.E. y GONZALES, A. Relación entre características del hábitat y estructura del ensamble de insectos en humedales palustres urbanos del centro-sur de Chile. Revista Chilena de Historia Natural, 2006, № 79, p.195-211.

VIDAL, C. y ROMERO, H. Efectos ambientales de la urbanización de las cuencas de los ríos Biobío y Andalién sobre los riesgos de inundación y anegamiento de la ciudad de Concepción. En: PÉREZ, L. e HIDALGO, R. Concepción metropolitano (AMC). Planes, Procesos y Proyectos. Santiago de Chile: GEOlibros, Pontificia Universidad Católica de Chile, 2010, p. 285-301.
VIÑALS, M. La variabilidad de las cubetas de los humedales mediterráneos: formas y procesos geomórficos. Humedales Mediterráneos, 1999, № 1, p. 91-98.

VITOUSEK, P.; ABER, J.; HOWARTH, R, LLIKENS, G.; MATSON, P.; SCHINDLER, D.; SCHLESINGER, W. \& TILMAN, G. Human alteration of the global nitrogen cycle: Sources and consequences. Ecological Applications, $1997, \mathrm{~N}^{\circ} 7, \mathrm{p}, 737-750$.

WOODROFFE, C. Coasts: form process and evolution. Cambridge: University Press, 2003.

YUAN, L.; GE, Z.; FAN, X. \& ZHANG, L. Ecosystem-based coastal zone management: A comprehensive assessment of coastal ecosystems in the Yangtze Estuary coastal zone. Ocean \& Coastal Management, 2014, No 95, p. 63-71.

ZHANG, L. \& SHAO, H. Direct planteplant facilitation in coastal wetlands: A review. Estuarine, Coastal and Shelf Science, 2013, N 119, p.1-6. 